\title{
Adult Intuitions about Mechanistic Content in Elementary School Science Lessons
}

\author{
Nicole Betz (nicole.betz@yale.edu) \\ Department of Psychology, Yale University \\ New Haven CT, 06520 USA
}

\author{
Amanda M. McCarthy (amanda.mccarthy@yale.edu) \\ Department of Psychology, Yale University \\ New Haven CT, 06520 USA
}

Frank C. Keil (frank.keil@yale.edu)

Department of Psychology, Yale University

New Haven CT, 06520 USA

\begin{abstract}
Elementary schools provide students with their first encounters with formal science, creating both foundations for students' knowledge of science content, and impressions of what it means to learn science. Here, we examined adult, including K12 teachers', intuitions regarding different types of content relevant to elementary school science, namely, labels, function, and mechanism. We focused primarily on perceptions of mechanistic explanations - causal explanations that explain how something works. This focus stems from children's curiosity and aptitude for mechanism. Across four studies we predicted, and found, that participants deprioritize mechanistic explanations relative to more superficial explanation types: labels and function. This intuition, which appears to be reflected in formal science curricula, misestimates children's abilities and attitudes towards mechanistic information.
\end{abstract}

Keywords: Explanations; Mechanistic Thinking; Science Learning; Children; Cognitive Development

\section{Introduction}

Adults often have a paradoxical impression of young children's capacities for learning. Children are simultaneously viewed as intellectual sponges able to quickly absorb new information, but also as immature minds that are unable to grasp complex concepts. These intuitions may influence how we interact with children, for example the types of explanations that we deem most appropriate for child-directed teaching.

In the context of science curricula, what do adults and teachers think should be the focus of these formal lessons? Should young children learn about internal mechanisms? Or should they start by developing a broad vocabulary of scientific terms? To address this question, we contrasted adults' beliefs surrounding the prioritization of superficial, nominal features of learning and deeper, mechanistic content in elementary school science across elementary school age groups. Such perceptions should reflect adults' estimation of developing cognitive capacities.

Young children certainly have cognitive limitations, such as reduced performance levels in tasks that impose heavy loads on memory and attention (Hambrick \& Engle, 2002). But memory and attentional deficits should not be equated with sweeping conceptual difficulties. Even infants have impressive foundational core capacities for cognitive reasoning (i.e., core knowledge; Spelke, 2000). Lay adults may underestimate such cognitive abilities in children even when considering the most basic cognitive processes. For example, adults erroneously believe that children lack the capacity for simple numerical discrimination (Wang \& Feigenson, 2019) and more complex cognition, such as understanding race (Sullivan, Wilton \& Apfelbaum, 2020). Even some cognitive developmental research appears to underestimate children's cognitive capacities by discounting the role of previous learning opportunities surrounding that topic (Duschl, Schweingruber \& Shouse, 2007). Such misestimations could impede opportunities for learning.

Elementary school science curriculum is an example of a formalized learning system at risk from misestimations of children's cognitive capacities. Indeed, the U.S. elementary science curriculum has been criticized for lacking explanatory depth, perhaps due to underestimating children's ability to learn more in-depth concepts (e.g., Duschl et al., 2007; Kelemen, 2019). Current U.S. elementary school science seems to focus on superficial content, such as briefly introducing a large number of topics (e.g., Schmidt, Wang \& McKnight, 2005), or developing children's vocabularies of scientific terminology (Snow, 2008). While terminology is important to learn, such superficial content can be taught simultaneously with richer, more satisfying explanations, such as mechanism (e.g., Betz \& Keil, under revision).

Although frequently omitted from elementary school lessons, the development and communication of mechanistic explanations are central to the scientific process. Mechanistic explanations break a phenomenon down into its component parts and describe how these parts interact to cause the phenomenon (Bechtel, 2011). These explanations answer "how" or "why" questions, and most directly, "How does that work". Mechanisms provide causally-rich information that resonates with young children's natural scientific curiosity (Kelemen 2019), fueling future scientific interest (Haeusler \& Donovan, 2020).

Moreover, emphasizing in-depth content (e.g., mechanism) over breadth in the classroom benefits future science learning. For example, students who covered at least one topic in depth in high school performed better in college level science courses than students whose curricula only 
focused on breadth (Schwartz et al., 2009). Similarly, elementary school children learn more after being given a mechanistic learning goal over more superficial goals (Betz $\&$ Keil, under revision). These and other findings (e.g., Haeusler \& Donovan, 2020) highlight the importance of teaching both mechanism and in-depth scientific explanations to children from an early age.

Despite these short- and long-term benefits of causal understandings, current educational practices in the classroom instead focus on providing students with superficial understanding of a breadth of scientific topics. Motivated by this incongruity, we examined lay adults' and teachers' perceptions of what types of explanatory content is appropriate from elementary-level science, thereby assessing lay intuitions about children's cognitive capacities for in-depth learning. In Studies 1 and 2, we assess intuitions regards the content currently included in elementary school lessons. In Studies 3 and 4, we assess intuitions regarding what should be included in lessons.

\section{Study 1}

Study 1 sampled lay adults and K-12 teachers, examining intuitions regarding the relative likelihood that three types of explanations (i.e., mechanism, function, labels) are taught to elementary school students. We predicted that adults would expect mechanistic explanations to be less likely to be taught, especially when considering younger learners.

\section{Method}

Participants. We recruited two samples of 180 adults via Amazon Mechanical Turk. One sample consisted of the general MTurk population (Age $M=36.5 ; 57$ female, 121 male, 2 non-binary), and the other consisted of K-12 teachers (Age $M=39.7 ; 115$ female, 65 male). A majority of the K-12 teachers reported teaching STEM courses (67.8\%), and a majority also reported teaching elementary school students (75.6\%). All participants were at least 18 years old, United States residents, and fluent English speakers.

Materials \& Design. Stimuli were short vignettes describing an elementary school teacher teaching her students various topics about science (e.g., "The science teacher is teaching her first-grade students about this microscope"). An image of a teacher standing next to the lesson topic (e.g., a microscope) accompanied each vignette.

In this 3 (grade) x 2 (domain) between-subjects design, participants were randomly assigned to one of six conditions that determined the content of these vignettes. These conditions varied in terms of the grade of the students in the teacher's classroom - either first, third, or fifth grade students - and the domain of scientific topics covered in the vignettes - either biological entities or artifacts.

Participant viewed eight vignettes, each representing a different topic. Participants rated the likelihood that the teacher would teach each of the three target scientific explanations within her lesson: 1) mechanistic (i.e., how the parts of the entity worked), 2) labels (i.e., what the parts of the entity are called), and 3) function (i.e., what functions the parts of the entity serve). Ratings were made on a scale ranging from 1 (Extremely Unlikely) to 6 (Extremely Likely).

\section{Results \& Discussion}

Data Coding. For each participant, we computed the average likelihood ratings for each of the three explanation types. (i.e., mechanism, labels, function).

Do Adults and Teachers think that Mechanism is Less Likely to be Taught? We predicted that adults, including K12 teachers, would expect less mechanistic explanations to be taught compared to other explanations. To test this, we computed a $2 \times 3 \times 2 \times 3$ mixed effects ANOVA on likelihood ratings, with population (K-12 teacher or lay adults) vignette age group (first, third, fifth) and item domain (biology, artifact) as between-subjects variables, and question type (mechanism, labels, function) as a within-subjects variable.

We found an overall effect of explanation type on likelihood ratings: $F(1.71,596.44)=28.78, p<.001$, et $a^{2}=.11$. Adults perceive mechanistic explanations to be significantly less likely to be taught in elementary schools $(M=4.6$, $S D=.99)$ than labels $(M=4.9, S D=.88)$ or functions $(M=4.8$, $S D=.86$ ).

This effect is qualified by a significant interaction between explanation type and domain: $F(1.71,596.44)=$ $7.42, p=.001$, eta $^{2}=.02$. The difference in likelihood ratings across conditions was stronger among participants considering lessons about biology than those considering lessons about artifacts. In both conditions, participants rated mechanistic explanations as less likely to be taught in lessons than both functions and labels (all Bonferroni $p$ 's $<.05)$. However, the larger effect when considering biology lessons suggests that adults, including teachers, may consider biological mechanisms to be especially omitted from elementary school science lessons.

We also found a main effect of age of students in vignette: $F(2,348)=7.58, p=.001$, et $a^{2}=.04$. Participants thought that all explanations were less likely when teaching first grade students $(M=4.5)$ than third $(M=4.8$; Bonferroni $p=.002)$ and fifth graders $(M=4.9$; Bonferroni $p=.003)$.

However, age of students in the vignettes did not interact with explanation type ( $p=.42$. Age also did not interact with domain $(p=.82)$, Thus, the preference for superficial explanations appears to persist across elementary school, regardless of topic.

Notably, there was no effect of population $(p=.29)$ and population did not interact with explanation type. The only effects of population were tangential to our research question (i.e., compared to the general population, teachers attributed higher likelihood ratings for all three explanation types when considering biology lessons). Thus, K-12 teachers and lay adults share the intuition that elementary school students are less likely to be taught mechanistic information in their science classes, particularly when considering lessons about biology. No other significant effects emerged. 


\section{Study 2}

Study 1 revealed that adults, including K-12 teachers, expected mechanistic explanations to be less present in elementary school science lessons compared to more superficial explanations (i.e., labels). Study 2 investigated a potential explanation for this intuition: the perceived difficulty of learning mechnaisms. We predicted that participants would judge mechanistic information to be more difficult to learn than other explanations, particularly for younger children. We further predicted that perceived difficulty would inversely predict likelihood ratings. Given the negligible difference in responses between K-12 teachers and the general MTurk population in Study 1, we henceforth sampled only from the general MTurk population.

\section{Method}

Participants. One hundred and eighty adults (89 male, 88 female, 2 nonbinary, 1 undisclosed; age $M=38.5$ ) participated via Amazon Mechanical Turk. All participants were at least 18 years old, resided in the United States, and were fluent English speakers.

Materials \& Design. The materials and design were identical to that of Study 1, with the addition of a second vignette block assessing perceived difficulty for students to learn each of the three scientific explanations. Participants thus rated each topic twice: for likelihood to teach and for difficulty to learn. Difficulty ratings were made on a scale ranging from 1 (Extremely Easy) to 6 (Extremely Difficult). The order of the likelihood and difficulty rating blocks was counterbalanced.

\section{Results \& Discussion}

Data Coding. For each participant, we computed the average likelihood ratings and difficulty ratings for each of the three explanation types (i.e., mechanism, labels, function).

Do Adults think that Mechanism is Less Likely to be Taught? We predicted that adults would again believe that mechanism is less likely to be taught in elementary school than other explanations. To test this, we computed a $3 \times 3 \times 2$ mixed effects ANOVA on likelihood ratings, with question type (mechanism, labels, function) as a within-subjects variable and vignette age group (first, third, fifth) and item domain (biology, artifact) as between-subjects variables.

We fully replicated all results from Study 1 . We again found a main effect of age group $[F(2,348)=6.34, p<.001$, $\left.e t a^{2}=.07\right]$, the main effect of explanation type, $[F(2$, $348)=33.67, p<.001$, eta $^{2}=.16$.] and the interaction between explanation type and domain $[F(2,348)=15.29, p<.001$, $e t a^{2}=.08$.], with the same patterns of results.

Do Adults Think Mechanism is More Difficult to Learn? We predicted that participants would perceive mechanistic information to be more difficult to learn than labels or functional explanations, particularly for younger children. To test this prediction, we computed a $3 \times 3 \times 2$ mixed effects ANOVA on difficulty ratings with explanation type (mechanism, labels, function) as a within-subjects variable and vignette age group (first, third, fifth) and item domain (biology, artifact) as between-subjects variables.

We found a main effect of explanation type: $F(2,348)$ $=64.47, \quad p<001, \quad e t a^{2}=.27$. As predicted, mechanistic explanations were seen as more difficult to learn $(M=3.9$, $S D=1.0)$ than either labels $(M=3.1, S D=1.0 ; t(179)=10.1$, $p<.001)$ or function $(M=3.5, S D=1.0 ; t(179)=6.4, p<.001)$. Further, functional explanations were seen as more difficult than labels: $t(179)=5.99, p<.001$.

Surprisingly, there was no interaction between explanation type and age $(p=.92)$. Thus, adults do not think that scientific explanations (e.g., mechanism) become easier for children to comprehend across elementary school.

Does Difficulty Predict Likelihood Ratings? We expected perceived difficulty to inversely predict likelihood ratings. In line with predictions, we found significant negative correlations between perceived difficulty ratings and likelihood ratings for all three explanation types: mechanism $[R h o(180)=-.460, p<.001]$, label $[R h o(180)=$ $-.395, p<.001]$, and function $[R h o(180)=-.430, p<.001]$. Moreover, we found inverse relationship $\left(p^{\prime} s<.03\right)$ when comparing perceived difficulty and likelihood for individual exemplars across all three explanation types, with the exceptions of two marginal inverse relationships and one non-significant inverse relationship (train mechanism; $p=.29$ ). This reveals a general tendency to believe that elementary school children are learning less difficult content.

To further explore the role of perceived difficulty to learn on likelihood for content to be taught in lessons, we computed a mediation analyses investigating perceived difficulty as a mediator between explanation type and perceived likelihood for content be taught. We used the Sobel test for mediation using the bootstrapping method (5000 resamples) with bias-corrected confidence estimates of coefficients (MacKinnon, Lockwood \& Williams, 2004). We used a dummy-coded categorical predictor variable for explanation type (i.e., mechanistic, labels, or function), which creates two paths surrounding the predictor variable - one comparing mechanistic and labels explanations $(M v L)$ and one comparing mechanistic and function explanations $(M v F)$.

For both $M v L$ and $M v F$ paths, perceived difficulty significantly mediated the relationship between explanation type and perceived likelihood. In the $M v L$ path, the indirect effect was .30, 95\% $C I[.20, .40]$. In the $M v F$ path, the indirect effect was $.14,95 \% C I[.05, .23]$. These indirect effects were reliably different from zero, indicating significant mediation (Dearing \& Hamilton, 2006). As evidenced by significance of direct effects, difficulty partially mediated the $M v L$ path $(\beta=.21, p=.02)$, but fully mediated the $M v F$ path $(\beta=.16, p=.06)$. 


\section{Study 3}

Studies 1 and 2 revealed that adults believe that children are less likely to be taught mechanistic explanations of scientific content. One factor contributing to this effect appears to be the perception that mechanism is more difficult for elementary students to learn than relatively superficial explanations.

In Studies 3 and 4, we explored what adults think elementary school students should be taught in science lessons, examining the type of content that adults prioritize. In Study 3, participants saw vignettes describing teachers who were pressed for time and forced to omit one type of explanation from their science lessons. We predicted that adults would select to cut the mechanistic explanations more than the labels or functional explanations when considering biological lessons. Given the relatively weaker differences in explanation ratings when considering artifacts in Studies 1 and 2, we did not have strong predictions about which would be cut when teaching about artifacts.

\section{Method}

Participants. One hundred and eighty adults (99 male, 81 female; age $M=36.91, S D=10.58$ ) participated via Amazon Mechanical Turk. All participants were at least 18 years old, United States residents, and fluent English speakers.

Materials \& Design. The materials were similar to those used in Study 1, apart from the following changes. First, Study 2 vignettes describe an elementary school science teacher who is short on time for her lesson (e.g., "The science teacher is teaching her first-grade students about this microscope. She only has time to teach two of the three explanations below).

Second, for each vignette, participants answered the following question instead of likelihood or difficulty ratings: which of the three following scientific explanations they think that the teacher should cut for time: 1) mechanistic, 2) label, and 3).The content of the explanation options were identical to those in Studies 1 and 2.

\section{Results \& Discussion}

Data Coding. For each participant, we summed the number of times (out of eight) they selected each explanation (i.e., mechanistic, label, and functional).

What Explanations do Adults Cut for Time? We expected adults who rated biology lessons to more often choose to cut mechanistic explanations when pressed for time. To test this, we computed a set of Chi Square analyses with explanation type (mechanism, labels, function), age (first, third, fifth), domain (biology, artifact), and the interactions thereof on decisions to cut from lessons.

Overall, decisions to cut differed by explanation type: $\chi^{2}(16)=46.08, p<.001$. Participants chose to cut mechanism from lessons $(M=3.4, S D=2.8)$ more than both labels
( $M=2.4, S D=2.8 ; Z=2.91, p<.001)$ or functions $(M=2.1$, $S D=2.4 ; Z=3.63, p<.001)$.

However, explanation type interacted with domain. The Chi Square comparing decisions to cut across explanations was only significant among participants considering lessons about biology: $\chi^{2}(16)=71.23, p<.001$ (artifacts $p=.29$ ). As predicted, participants who read vignettes about biology lessons were more likely to choose to cut mechanistic explanations compared to labels $(Z=4.67 ; p<.001)$ or function $(Z=3.69 ; p<.001$; see Figure 1$)$, while participants who read vignettes about artifacts cut all three explanations more equitably (all $p$ 's $>.18$ ).

These findings suggest that adults deprioritize mechanistic explanations when teaching elementary school children about biological entities, instead prioritizing more superficial content like labels or function.

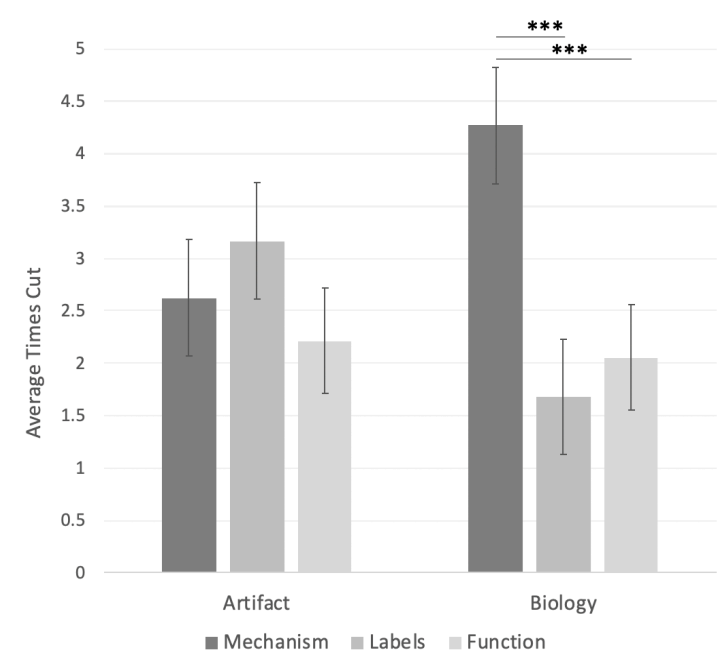

Figure 1: Effects of Vignette Domain on Choice of Explanation to Cut for Time. Error bars represent 95\% confidence intervals. $* * * p<.001 .+p<.10$.

\section{Study 4}

Mechanistic explanations are one example of how teachers can provide in-depth explanations of a scientific entity. Thus, in Study 4, we assessed whether adults believe that elementary school children should be taught broad, superficial lessons that teach several labels or focused, indepth lessons about a mechanistic process. We predicted that participants would believe that it is more important for elementary school students to be briefly introduced to a large number of topics in their science classes (i.e., superficial breadth) than to be taught a comprehensive explanation about how one scientific process work (i.e., mechanistic depth).

\section{Method}

Participants. One hundred and eighty adults, (94 male, 84 female, 1 nonbinary, 1 undisclosed; age $M=36.53, S D=$ 10.56) participated via Amazon Mechanical Turk. All 
participants were at least 18 years old, United States residents, and fluent English speakers.

Materials \& Design. The main testing block contained a set of short vignettes asking participants to imagine that they are a member of the Parent Teacher Association and that an elementary school teacher is asking for their input on which lessons to teach. For each of eight topics, participants rated how likely they would be to recommend that the teacher teaches each of two lessons. Both lessons are summarized by descriptive titles that highlight that the lessons focus on mechanistic depth (e.g., How Does That Work? The Way Mushroom Parts Work Together) or superficial breadth (e.g., What is That Called? The Mushroom and Other Fungi). As in this example, the lesson options focusing on superficial breadth would teach the labels of a series of related entities, while the lesson option focusing on mechanistic depth would teach a mechanistic process within one entity. Ratings were made on a scale ranging from 1 (Extremely Unlikely to Recommend) to 6 (Extremely Likely to Recommend).

After answering questions for all eight vignettes, participants rated the general importance of teaching both of the following types of content in the science classes: "a comprehensive explanation about how a small number of topics work," and "a brief introduction to a large number of topics." The order of these questions was counterbalanced. Ratings were made on a scale ranging from 1 (Extremely Unimportant) to 6 (Extremely Important).

Participants were randomly assigned to one of six experimental conditions that determined the topics and grades of children within the vignettes and the grade specified in the importance question in a 3 (grade: first, third, fifth) x 2 (domain: biology, artifact) between-subjects design.

\section{Results and Discussion}

Data Coding. For each participant, we computed the average rated likelihood to recommend and importance of both of the information types (i.e., mechanistic depth, superficial breadth).

Do Adults Recommend Lessons Focusing on Superficial Breadth? We predicted that participants would be more likely to recommend elementary school lessons focusing on superficial breadth over mechanistic depth. To test this, we computed a $2 \times 3 \times 2$ mixed effects ANOVA on likelihood ratings with information type (mechanistic depth, superficial breadth) as a within-subjects variable and vignette age group (first, third, fifth) and item domain (biology, artifact) as between-subjects variables. We found a main effect of information type on recommendation likelihood ratings: $F(1,174)=9.94, p=.002$, et $a^{2}=.05$. As predicted, participants were more likely to recommend lessons focused on superficial breadth $(M=4.5, S D=1.0)$ over mechanistic depth $(M=4.2, S D=1.1)$.

This effect was qualified by a significant interaction between information type and age: $F(1,174)=9.15, p<.001$, et $a^{2}=10$. Participants who viewed vignettes about first graders showed the predicted preference for lessons focusing on superficial labels $(p<.001)$, but this preference decreased as the age of the students in the vignette increased. Participants who viewed vignettes about third graders showed a marginal preference for superficial labels $(p=.08)$, while participants who viewed vignettes about fifth graders showed no preference $(p=.20)$. These data partially support our prediction that adults prioritize superficial lessons in elementary science classes, with the caveat that this preference decreases across elementary school. This pattern helps to explain why young elementary school children may be taught to learn science by focusing on developing a scientific vocabulary, rather than focusing on developing a foundation for mechanistic thinking.

Finally, we found a significant effect of domain on recommendation likelihood ratings: $F(2,174)=5.52, p=.02$, $e t a^{2}=.03$. Overall, adults were more likely to recommend lessons focused on biological content $(M=4.47, S D=.81)$ than lessons focused on artifact content $(M=4.18, S D=.88)$. This suggests that adults may deprioritize engineering or technological-focused content in the science classroom.

Do Adults Think Superficial Breadth is More Important for Elementary School Science? We predicted that adults would report that it is more important for elementary school students to be taught superficial breadth than mechanistic depth. To test this, we computed a $2 \times 3 \times 2$ mixed effects ANOVA on likelihood ratings with content type (mechanistic depth, superficial breadth) as a withinsubjects variable and vignette age group (first, third, fifth) and item domain from main testing block (biology, artifact) as between-subjects variables. We found a main effect of information type: $F(1,174)=21.39, p<.001, e t a^{2}=.11$. As predicted, participants thought that it was more important for elementary school lessons to teach superficial breadth $(M=4.8, S D=1.2)$ than mechanistic depth $(M=4.2, S D=1.3)$.

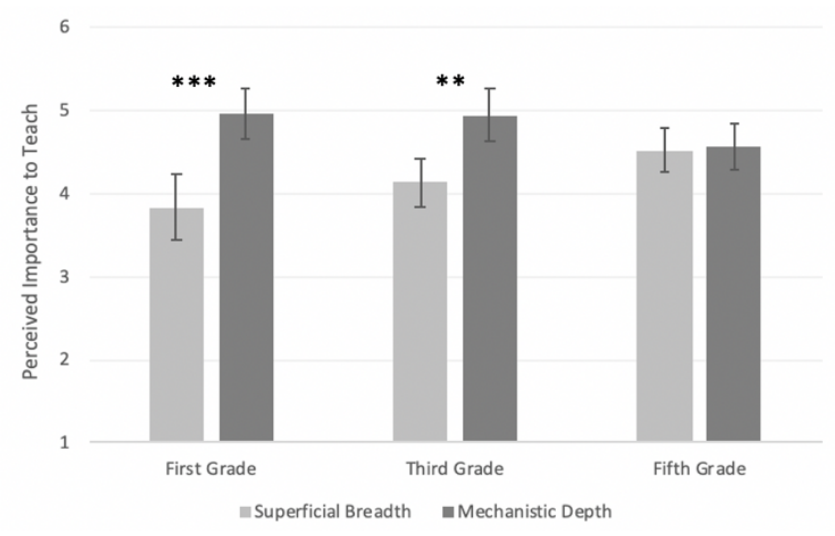

Figure 2: Adults' perceived importance to teach superficial breadth or mechanistic depth in elementary school classes, across student age groups. Error bars represent 95\% confidence intervals. ${ }^{* * *} p<.001 .{ }^{* *} p<.01$. 
This effect was qualified by a significant interaction between age and information type: $F(2,174)=4.98, p=.008$, et $a^{2}=.05$ (see Figure 2). Importance ratings for mechanistic depth were significantly higher than superficial breadth for participants who read vignettes about either first (breadth $M=4.95$, depth $M=3.83$; Bonferroni $p<.001$ ) or third grade students (breadth $M=4.93$, depth $M=4.13$; Bonferroni $p=.002$ ). Importance ratings did not differ among those who read about fifth grade students $(p=.81)$ Further, mechanistic depth was perceived as more important when considering fifth grade students than first graders $(t(118)=2.87, p=.005)$. Thus, adults' prioritization of breadth over mechanistic depth decreases across elementary school age groups.

\section{General Discussion}

Even though lay adults shape children's learning of science by assisting with their formal and informal education, little is known about adults' intuitions about the content of elementary school education. Such intuitions can speak to adults' misestimations of children's learning abilities and preferences. We investigated adults' descriptive beliefs about the presence of mechanistic content (Studies 1 and 2) as well as beliefs surrounding what content should be prioritized (Studies 3 and 4) in elementary school classrooms. More specifically we asked: What intuitions do adults have regarding mechanism in elementary school science? and Do adults prioritize superficial breadth over mechanistic depth?

\section{Adults expect less mechanism in elementary lessons}

Lay adults and K-12 teachers alike expect mechanistic explanations to be relatively uncommon in elementary school science classrooms. Notably, this bias does not just describe perceptions of content taught in current classrooms but extends to adults' beliefs of what should be prioritized. Adults more often choose to cut mechanistic content, rather than function or labels, from lessons when pressed for time.

To the extent that these perceptions accurately reflect current elementary school science classes, this bias against mechanism contradicts overt guidelines from the Next Generation Science Standards (2013) that emphasize the importance of teaching more in-depth explanations across the K-12 science curriculum. Limiting mechanistic explanations in elementary schools means avoiding teaching children the explanations that they find most important and interesting, potentially shaping expectations of science as a collection of dissatisfying, superficial content (e.g., terminology).

Indeed, elementary-aged children are quite curious about how things work (e.g., Mills et al., 2011) and teaching mechanism enhances science interest (Hauseler \& Donovan, 2020). If these mechanistic curiosities are not satisfied in science classrooms, students may grow frustrated. Over time, these frustrations may contribute to longer-term disenchantment towards formal science if they expect future science lessons to be similarly superficial and unsatisfying.

Further, adults perceived mechanistic content to be relatively difficult for children to understand in elementary school science, particularly in the younger years (i.e., first grade). This perceived difficulty to learn mediated the relationships between explanation types and perceived likelihood for explanations to be taught. Adults appear to think that mechanistic explanations are not taught in elementary school classrooms in large part because the content is too difficult for children to learn.

Notably, however, young children are able to successfully learn and understand mechanistic explanations even prior to elementary school. Children as young as three can have rudimentary mechanistic understandings of simple machines, like gear trains (e.g., Metz, 1991) and children as young as five can understand abstract causal principles of evolutionary inheritance (e.g., Kelemen et al., 2014). Thus, mechanistic explanations can be successfully tailored to young children.

Adults' bias against mechanism may be linked to a preference to teach young children purportedly easy, superficial information such as labels. However, these goals need not compete with each other. Children can glean information about superficial details (e.g., names of parts of an entity) from learning more satisfying mechanistic stories (e.g., Betz \& Keil, under revision). This approach could allow children to learn more cursory information in a way that children find more interesting and explanatorily satisfying.

Interestingly, there were stark differences in explanation preferences across domains. While adults and K-12 teachers consistently deprioritized mechanistic information about biology, this effect was less consistent when considering artifacts. This may reflect stronger associations among adults between mechanisms and artifacts, or perceptions that teachers may not have the foundational knowledge to teach biological mechanisms. Indeed, almost a third of elementary school teachers express low confidence in their ability to teach science to children (Banilower, 2019).

\section{Adults prioritize breadth over mechanistic depth}

Adults generally prioritized superficial breadth over mechanistic depth when considering elementary school science lessons. As children mature, adults transitioned towards focusing on both depth and breath.

This shift in prioritization from breadth towards mechanistic depth aligns with the timing of the welldocumented decreased interest in formal science that occurs around fifth grade (Potvin \& Hasni, 2014). One explanation for this declining interest is a shift in the complexity and difficulty of content taught in science classes from elementary to middle schools (Kelemen, 2019). By teaching children scientific mechanism earlier in elementary school, educators may be able to better prepare students to learn more detailed, complex mechanistic information later on (Kelemen, 2019). Moreover, teaching in-depth mechanisms can create a foundational understanding of science as a process of developing formal causal understandings.

\section{Implications for Intuitions about Explanations}

Although our tasks directly assess intuitions regarding teaching science to elementary school students, our findings 
may shed some light on adults' intuitions about explanations more broadly. In particular, adults preferred superficial labels over more in-depth explanations such as mechanism or functions. While we inquired about child-directed explanations, this preference accords with perceptions that explanations containing labels are more explanatorily satisfying (Griffin, Wilkenfeld \& Lombrozo, 2017), and adult preferences for explanations with labels even when the labels provide no additional explanatory information (Hemmatian \& Sloman, 2018). Indeed, the perceived explanatory utility of labels may explain adults' partiality for teaching labels to elementary school children. Thus, our effects may result from an interaction of erroneous intuitions: the overestimation of explanatory power of labels, the perceived difficulty of mechanistic content, and the underestimation of children's abilities. In the context of science education, these intuitions may delay the introduction of satisfying, in-depth mechanistic explanations about science.

While adults and instructors might be trying to strategically choose lessons that they believe are better suited to their students' abilities, they may be building educational expectations for superficial information that do not persist beyond elementary school. If educators can avoid a bias against teaching mechanism, they have an opportunity to put students on a mechanism-rich science trajectory, fostering scientific enjoyment and future science learning.

\section{Acknowledgments}

We thank all lab members for feedback on preliminary versions of these studies. This research was funded by NSF \#DRL 1561143 granted to Frank Keil.

\section{References}

Banilower, E. R. (2019). Understanding the big picture for science teacher education: The 2018 NSSME+. Journal of Science Teacher Education, 30(3), 201-208.

Bechtel, W. (2011). Mechanism and biological explanation. Philosophy of science, 78(4), 533-557.

Betz, N. \& Keil, F., (under revision). Mechanistic Mindset: A Mechanistic Focus Benefits Elementary Student. CogSci

Dearing, E., \& Hamilton, L. C. (2006). Contemporary advances and classic advice for analyzing mediating and moderating variables. Monographs of the society for research in child development.

Duschl, R. A., Schweingruber, H. A., \& Shouse, A. W. (Eds.). (2007). Taking science to school: Learning and teaching science in grades K-8 (Vol. 500). Washington, DC: National Academies Press.

Giffin, C., Wilkenfeld, D., \& Lombrozo, T. (2017). The explanatory effect of a label: Explanations with named categories are more satisfying. Cognition, 168, 357-369

Haeusler, C., \& Donovan, J. (2020). Challenging the science curriculum paradigm: Teaching primary children atomicmolecular theory. Research in Science Education, 50(1), 23-52.

Hambrick, D. Z., \& Engle, R. W. (2002). Effects of domain knowledge, working memory capacity, and age on cognitive performance: An investigation of the knowledge-is-power hypothesis. Cognitive psychology, 44(4), 339-387.

Hemmatian, B., \& Sloman, S. A. (2018). Community appeal: Explanation without information. Journal of Experimental Psychology: General, 147(11), 1677.

Kelemen, D. (2019). The magic of mechanism: Explanation-based instruction on counterintuitive concepts in early childhood. Perspectives on Psychological Science, 14(4), 510-522.

Kelemen, D., Emmons, N. A., Seston Schillaci, R., \& Ganea, P. A. (2014). Young children can be taught basic natural selection using a picture-storybook intervention. Psychological science, 25(4), 893-902.

MacKinnon, D. P., Lockwood, C. M., \& Williams, J. (2004). Confidence limits for the indirect effect: Distribution of the product and resampling methods. Multivariate behavioral research, 39(1), 99-128.

Metz, K. E. (1991). Development of explanation: Incremental and fundamental change in children's physics knowledge. Journal of Research in Science Teaching, 28(9), 785-797.

Mills, C. M., Legare, C. H., Grant, M. G., \& Landrum, A. R. (2011). Determining who to question, what to ask, and how much information to ask for: The development of inquiry in young children. Journal of Experimental Child Psychology, 110(4), 539-560.

NGSS Lead States. (2013a). Next Generation Science Standards: For states, by states (vol. 1, The Standards). Washington, DC: The National Academies Press.

Potvin, P., \& Hasni, A. (2014). Analysis of the decline in interest towards school science and technology from grades 5 through 11. Journal of Science Education and Technology, 23(6), 784-802.

Schmidt, W. H., Wang, H. C., \& McKnight, C. C. (2005). Curriculum coherence: An examination of US mathematics and science content standards from an international perspective. Journal of curriculum studies, 37(5), 525-559.

Schwartz, M. S., Sadler, P. M., Sonnert, G., \& Tai, R. H. (2009). Depth versus breadth: How content coverage in high school science courses relates to later success in college science coursework. Science education, 93(5), 798-826.

Snow, C. (2008). Essay: What is the vocabulary of science. Teaching science to English language learners: Building on students 'strengths, 71-84.

Spelke, E. S. (2000). Core knowledge. American psychologist, 55(11), 1233.

Sullivan, J., Wilton, L., \& Apfelbaum, E. P. (2020). Adults delay conversations about race because they underestimate children's processing of race. Journal of Experimental Psychology: General.

Wang, J., \& Feigenson, L. (2019). Infants recognize counting as numerically relevant. Developmental science, 22(6), e12805. 